\section{Vom Wert der Natur}

Nie war der Verlust biologischer Vielfalt, die Zerstörung der Wälder, der Meere und der Böden so massiv wie heute. Und trotzdem ist Naturschutz unpopulär. Ökonomische Argumente sollen dem Naturschutz zum Erfolg verhelfen. Kann das gut gehen? Von Barbara Unmüßig

$\mathbf{N}$ aturschutz hat kaum eine politische Lobby. Der große Trend: Es fehlt am politischen Willen der Regierungen, dem Natur- und Ökosystemschutz eine politische Priorität einzuräumen. Klassische Naturschutz- und Biodiversitätspolitik - auf nationaler wie internationaler Ebene - stößt an ihre Grenzen und es fehlt überall an Geld für den Biodiversitätsschutz. In diesem Kontext argumentieren Umwelt- und Naturschützer, unterstützt von Umweltökonomen, für eine ökonomischere Sichtweise auf Natur und für mehr marktbasierte Instrumente im Naturschutz.

Gestattet sei die Frage, ob diese Form der volkswirtschaftlichen Bereitstellung von Information, Politik zum Umsteuern bewegt. Denn dieser Begründung liegt die bemerkenswerte Annahme zugrunde, dass sich ökonomische (Macht-) Interessen, die der Zerstörung von Ökosystemen zugrunde liegen, mit Informationen aushebeln ließen.

\section{Bewertung statt Willensbildung?}

Die ökonomische Bewertung von Natur ist allerdings sinnvoll und nützlich, wenn monetäre Kompensationen für die Zerstörung von Natur berechnet werden sollen. Irreversible Schäden können allerdings so nicht kompensiert werden. Der Primat der Politik bleibt also herausgefordert. Der Schutz der Biodiversität braucht vor allem politische Willensbildung und starke gesellschaftliche Allianzen.
Die Sichtbarmachung und Berechnung von Dienstleistungen der Natur ist längst Praxis und mündet in Zahlungen für Ökosystemschutz (Payments for Ecosystem Services). Der Schutz beziehungsweise umweltverträgliches Wirtschaften ist bei einem seit Langem fest verankerten Prinzip der Kompensationsund Ausgleichszahlungen nicht in gleichem Maße der Fall. Das Versprechen hier ist, den Verlust von Biodiversität durch wirtschaftliche Eingriffe durch den Schutz von Biodiversität an anderer Stelle zu kompensieren (Offsetting). Ein Kompensations- und Ausgleichsmechanismus soll nun im Rahmen der EU-Biodiversitätsstrategie 2020 ausgebaut werden. Das heißt dann No-Net-Loss-Initiative. Ein EU-weites Gesetz soll es 2015 geben.

Ob diese Initiative eher schadet als nützt, wird zu Recht heftig diskutiert. Mit Offsetting wird suggeriert, es ließe sich Biodiversität an anderer Stelle reproduzieren. Lokal Gebundenes wie Biodiversität und eigentlich Ungleiches, Diverses wird ökonomisch vergleichbar gemacht. Und: Die Bezahlung von Naturerhalt wird an deren Zerstörung gekoppelt. Und zwar nicht als auszuhandelnde Maßnahme, sondern als regulären Mechanismus. Offsetting fördert so die Legitimation und Akzeptanz von Zerstörung und generiert zudem eine problematische Pfadabhängigkeit für die Finanzierung von Naturschutz. Eine Falle, in der der deutsche Naturschutz längst sitzt.

Eine weitere und neuere Variante im Naturschutz ist, aus einzelnen Ökosys- temdienstleistungen ein handelbares Gut zu machen. Sie werden monetarisiert und sollen $\mathrm{zu}$ einer profitablen Quelle für den Naturschutz und den Privatsektor werden. Bedeutende Akteure wie die Weltbank und einzelne Regierungen arbeiten an deren Umsetzung. Die Bezahlung der Umweltleistung ist auch an die Logik des Offsettings geknüpft. Offsetting ist in den meisten Fällen die Kehrseite der Monetarisierung von Umweltleistung.

\section{Natur als Wirtschaftsakteur?}

Einzelne Dienstleistungen der Natur wie die Speicherung von Kohlendioxid durch einen Wald in Geldwert zu setzen, heißt, die komplexen sozialen, ökologischen und kulturellen Funktionen von Ökosystemen zu ignorieren und sie ihres sozialen und ökologischen Kontextes zu berauben. Die sozialen und gesellschaftlichen Mensch-, Natur- und Machtverhältnisse werden neu strukturiert, besonders dort, wo plötzlich natürliche Dienstleistungen privatisiert werden.

„You cannot manage what you don't measure“ - diesem Ehrfurcht gebietenden Glaubenssatz sollten wir uns nicht immer anschließen. Natur ist eben kein Wirtschaftsakteur. Die Wertschätzung der Natur braucht uns alle. Ökonomische Berechnungen können hilfreich und sinnvoll sein. Wir sollten aber Grenzen dort ziehen, wo die weitere Ökonomisierung der Natur die Lebensgrundlagen von Menschen und letztlich politisches Handeln untergräbt, weil nur noch als schützenswert gilt, was ökonomisch messbar ist, statt sich dem Allgemeinwohl und allen Funktionen der Natur zu verpflichten.

AUTORIN + KONTAKT Barbara Unmüßig ist Vorstand der Heinrich-Böll-Stiftung in Berlin.

Heinrich-Böll-Stiftung, Sekretariat Vorstandsbüro Barbara Unmüßig, Kathrin Klaua, Schumannstraße 8, 10117 Berlin. Tel. +49 30 28534114, E-Mail: klaua@boell.de vates License (http://creativecommons.org/licenses/by-nc-nd/4.o/deed.de), which permits copying and redistributing the material in any medium or format, provided the original work is properly cited, it is not used for commercial purposes and it is not remixed, transformed or built upon. 